\title{
Dynamic Process Migration Based on Block Access Patterns Occurring in Storage Servers
}

\author{
JIANWEI LIAO, Southwest University, China \\ FRANÇOIS TRAHAY, Université Paris Saclay, France \\ GUOQIANG XIAO, Southwest University, China
}

\begin{abstract}
An emerging trend in developing large and complex applications on today's high-performance computers is to couple independent components into a comprehensive application. The components may employ the global file system to exchange their data when executing the application. In order to reduce the time required for input/output (I/O) data exchange and data transfer in the coupled systems or other applications, this article proposes a dynamic process migration mechanism on the basis of block access pattern similarity for utilizing the local file cache to exchange the data. We first introduce the scheme of the block access counting diagram to profile the process access pattern during a time period on the storage server. Next, we propose an algorithm that compares the access patterns of processes running on different computing nodes. Last, processes are migrated in order to group processes with similar access patterns. Consequently, the processes on the computing node can exchange their data by accessing the local file cache, instead of the global file system.

The experimental results show that the proposed process migration mechanism can reduce the execution time required by the application because of the shorter I/O time, as well as yield attractive I/O throughput. In summary, this dynamic process migration technique can work fairly well for distributed applications whose data dependency rely on distributed file systems.
\end{abstract}

Categories and Subject Descriptors: D.4.3 [File Systems Management]: Distributed file systems

General Terms: Design, Algorithms, Performance

Additional Key Words and Phrases: Distributed file system, block access events, access counting diagram, pattern similarity, process migration, I/O performance

ACM Reference Format:

Jianwei Liao, François Trahay, and Guoqiang Xiao. 2016. Dynamic process migration based on block access patterns occurreing in storage servers. ACM Trans. Archit. Code Optim. 13, 2, Article 20 (June 2016), 20 pages.

DOI: http://dx.doi.org/10.1145/2899002

\section{INTRODUCTION}

High-performance clustersand other high-performance distributed computer systems, which consist of computing nodes for executing processes and storage nodes

This work was partially supported by the National Natural Science Foundation of China (Grant No. 61303038); the Natural Science Foundation Project of CQ CSTC (Grant No. CSTC2013JCYJA40050); the Scientific Research Foundation for the Returned Overseas Chinese Scholars, State Education Ministry; and the Special Program for Applied Research on Super Computation of the NSFC-Guangdong Joint Fund (the second phase).

Authors' addresses: J. Liao and G. Xiao, College of Computer and Information Science, Southwest University of China; email: gqxiao@swu.edu.cn; F. Trahay, CNRS Samovar, Télécom SudParis, Université Paris Saclay, France; email: francois.trahay@telecom-sudparis.eu. Corresponding author: J. Liao, liaotoad@gmail.com. He is now an associate professor at Southwest University of China.

Permission to make digital or hard copies of part or all of this work for personal or classroom use is granted without fee provided that copies are not made or distributed for profit or commercial advantage and that copies show this notice on the first page or initial screen of a display along with the full citation. Copyrights for components of this work owned by others than ACM must be honored. Abstracting with credit is permitted. To copy otherwise, to republish, to post on servers, to redistribute to lists, or to use any component of this work in other works requires prior specific permission and/or a fee. Permissions may be requested from Publications Dept., ACM, Inc., 2 Penn Plaza, Suite 701, New York, NY 10121-0701 USA, fax +1 (212) 869-0481, or permissions@acm.org.

(c) 2016 ACM 1544-3566/2016/06-ART20 $\$ 15.00$

DOI: http://dx.doi.org/10.1145/2899002 
for providing input/output (I/O) services, are widely used to solve large scientific applications [Vecchiola et al. 2009]. However, due to the increase of application scale and of the data size, one of the most critical challenges to be addressed is about how to overcome the data access bottleneck [Dongarra et al. 2011].

Specifically, the noticeable issue arises from the study area of data I/O in highperformance computing: The I/O transfer time needed for exchanging data among processes belonging to the same application depends on both the affinity of the processes and the locations of processes [Jeannot et al. 2014]. That is to say, if two processes are located on the same node, then both of them can exchange data by using the local file cache. As a consequence, the time required for exchanging data by using the local file can be considerably reduced, compared to exchanging the data via the parallel file system.

In other words, preserving data locality by placing the computation tasks close to their required data is crucial for performance in large clusters, because the slow data rate on the disk and the network bisection bandwidth become a bottleneck [Dean and Ghemawat 2008]. In order to reduce the cost of inter-process communication (i.e., Message Passing Interface (MPI) communication [MPI 2012]), many studies have focused on binding processes to the proper computing nodes [Chen et al. 2006; Mercier and Jeannot 2011]. These mechanisms make sense if and only if the binding process knows the communication pattern of the application (i.e., which process communicates with which process).

However, many problems in science and engineering can be solved by coupling interacting models, which may result in several independent/semi-independent models for handling the different steps. For example, in modeling long-term global climate, the Community Climate System Model (CCSM) includes an atmosphere model, an ocean model, a sea-ice model, and a land-surface model [Hack et al. 2006]. On the other side, these models have been separately developed by different researchers, thus, they may present distinct behaviors deriving from their interdisciplinary essence and from their computation flow in the algorithms [Larson et al. 2005; Valcke et al. 2012]. As a result, the processes belonging to the different models do not have the information about where to send/get their output/input data. For this reason, this kind of application commonly leverages the global file system for exchanging the data. But, disk-based data I/O operations not only slow down the execution of the application but also place pressure to the file system.

This article proposes a mechanism that analyzes the I/O transfers of a distributed application and dynamically migrates processes between computing nodes, for the purpose of improving the locality of I/O accesses. Therefore, instead of using the distributed file system, processes can exchange data via the local file cache on the computing node, which can reduce the cost of data transfers. In this mechanism, we first employ the newly proposed scheme of block access counting diagram to profile the access pattern on the storage server for each process; then we compute the access pattern similarity of two processes. Depending on their similarity, some of the processes may then be dynamically migrated to other computing nodes in order to enhance the locality of I/O accesses and to yield a better system performance. In brief, this article makes the following two contributions:

(1) On the storage server side, we propose the techniques of block access counting diagram and $N$-compressed access counting diagram to classify block access patterns in a certain period with a per-process granularity. The access patterns are then compared in order to estimate the pattern similarity of two processes.

(2) We also propose a novel scheme to perform process migration based on the similarity of access patterns. This migration strategy groups processes with similar access 
patterns together to optimize the data locality and thus maximize the usage of the local file cache. As a consequence, besides accelerating the I/O operations, the data rate of the $\mathrm{I} / \mathrm{O}$ subsytem is greatly improved.

The rest of the article is structured as follows: The related work regarding process migration is described in Section 2. The design and implementation details of this newly proposed process migration mechanism are illustrated in Section 3. Section 4 introduces the evaluation methodology and discusses experimental results. At last, we make concluding remarks in Section 5.

\section{RELATED WORK}

Many previous studies have focused on process migration, block access pattern-based I/O optimization, and the coupling tools for independent models:

Dynamic Process Migration: The mechanism of dynamic process migration by using the checkpoint/restart facility is widely used for a variety of purposes:

(1) Dynamic processes migration for balancing workloads. C. Du and X. Sun et al. proposed a dynamic scheduling mechanism that takes migration cost and other conventional influential factors into account to boost system performance in a shared, heterogeneous environment [Du et al. 2007]. L. Pilla and C. Ribeiro et al. have introduced a novel hierarchical load-balancing mechanism by adopting dynamic process migration, to enhance the performance of applications on parallel multi-core systems [Pilla et al. 2012].

(2) Dynamic processes migration for achieving fault tolerance. Checkpoint/restart is the most popular technique in high-performance computing to offer high availability and reliability. It is simple and effective enough in situations where the failures do not occur frequently. The fundamental principle of checkpoint/restart is to periodically save the state of the target process. If, for some reason, the process crashes, then the most recent checkpointed state is read and the process execution restarts from there [Liao 2012]. Although the idea of checkpoint/restart is quite straightforward, it adopts many variants in the specified application contexts [Meneses et al. 2015; Dong et al. 2011].

Especially, with the emerging of virtualization techniques [Williams et al. 2012], various virtual machine replication and migration approaches have been introduced. $\mathrm{L}$. Cheng and C. Wang have proposed a new I/O virtualization approach called vBalance to substantially boost the I/O performance for Symmetric MultiProcessing (SMP) virtual machines [Cheng and Wang 2012]. This approach is a cross-layer solution to speedup I/O operations by migrating interrupts from a preempted vCPU to a running one. Through migrating operating systems running services with liveness constraints in a virtual machine, it is possible to rapidly move the interactive workloads within clusters and data centers and then reach the targets of load balancing and fault tolerance [Clark et al. 2005].

In addition, in order to enhance the communication performance among the MPI processes in multi-core clusters, E. Jeannot and G. Mercier et al. have proposed algorithms that place MPI processes on computing nodes according to their communication patterns. That is to say, the processes that communicate a lot with each other are allocated on the same or nearby nodes. The overhead of inter-process MPI communication is thus greatly decreased [Mercier and Jeannot 2011; Jeannot et al. 2014]. P. Michaud et al. have proposed a thread migration mechanism that is able to maximize system performance for ensuring the necessary thread migrations on the basis of a temperature constraint [Michaud et al. 2007]. Q. Chen and M. Guo have presented an adaptive scheme of task scheduling on the basis of workloads for 
asymmetric multicore architectures [Chen and Guo 2014]. There are also some tools for profiling the MPI communications at runtime to offer the basic information to perform possible optimization [Tikir et al. 2009; Fuerlinger et al. 2010].

Active Storage Systems and Other Intelligent Storage Systems: The active storage system is aimed at I/O bound applications that involve fundamentally independent data sets [Piernas-Canovas and Nieplocha 2010]. It takes advantage of the underutilized computing resources in the storage servers and supports migrating the processes from computing nodes to the proper storage nodes [Piernas et al. 2007; Xie et al. 2016]. As a result, the processes can be executed on the storage nodes by using a large amount of data without any data transfers between the computing nodes and storage nodes, for achieving better system performance. However, the migrated processes are likely to use certain restricted kernel functions offered by the computing nodes in some cases, and, because of resource limitations on storage nodes, it might be impossible to execute this kind of tasks on the storage nodes. Furthermore, direct access to the storage nodes is not generally allowed in real-world production environments for security reasons [Zheng et al. 2013].

To offer intelligent storage services for different I/O intensive workflows, L. Costa et al. have presented a mechanism to enable making runtime system configuration decisions, including chunk size and caching policies, in a storage system to speedup $\mathrm{I} / \mathrm{O}$ processing in the case of specified application context [Costa et al. 2014]. Z. Gong have introduced a high-performance parallel I/O middleware for large-scale HPC applications to yield user-transparent layout optimization for scientific workflows, on the basis of access patterns of the applications [Gong 2013].

Block Access Patterns-Based I/O Optimization: In order to yield better I/O performance, there are also substantive optimization strategies that employ the information about block access patterns on the storage nodes or the local file server. $\mathrm{Z}$. Li and Y. Zhou et al. proposed a data-mining approach called $C$-miner to explore block correlations in the file server on a local machine so the file system can make use of the discovered block correlations for guiding I/O optimization strategies, such as data prefetching or data movement [Li et al. 2004]. Similarly, S. Jiang et al. have implemented DiskSeen, which employs a frequent sequence-based pattern modeling technique to classify block access pattern, and both temporal and spatial correlations of block access events have been taken into account for improving the sequentiality of disk accesses and overall prefetching performance [Ding et al. 2007; Jiang et al. 2013]. J. He et al. have proposed file re-organization on the basis of logical I/O patterns, which occur in the computing nodes for expediting the I/Os [He et al. 2013].

Moreover, we have proposed and implemented a server-side prefetching mechanism in distributed file systems in our previous work [Liao et al. 2015], which takes into account the regularity of the block access events that occur on storage servers. This I/O optimization scheme utilizes the block access patterns to direct prefetching data on the storage nodes. Then the prefetched data are proactively forwarded to the client node to speed up the execution of the applications running on the resource-limited computing nodes (i.e., client nodes) and to boost I/O data throughput.

Coupling mechanisms for separated models or applications. The Model Coupling Toolkit (MCT) [Larson et al. 2005] is a library providing routines and datatypes for creating a coupled system and mainly used in the CCSM. Hereafter, S. Valcke et al. [2012] have summarized major coupling technologies used in Earth System Modeling, and their article shows common features of the existing coupling approaches including the functionality to communicate and re-grid data. Moreover, the OASIS coupler is another typical related work that is able to process synchronized exchanges of coupling information generated by different components of the climate system, and the separate coupler mediates communication among the components [Valcke et al. 2006]. However, 


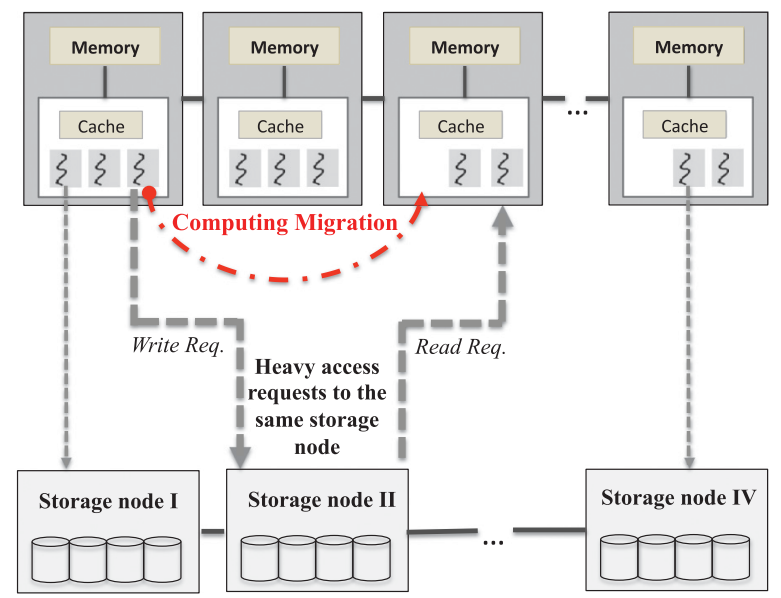

Fig. 1. The illustration of dynamic process migration on the basis of data dependency. (Note: Data dependency can be represented with block access pattern similarity.)

these coupling tools require us to modify the source codes of applications to use their specific interfaces. That is to say, they cannot keep the transparency to the application users and fail to offer a general solution to all cases.

\section{DESIGN AND IMPLEMENTATION}

We propose a novel mechanism that dynamically migrates processes from one computing node to another to effectively use the local file cache on the computing node. The decision to migrate a process to another computing node is done according to the similarity of the block I/O access patterns of the computing processes.

Figure 1 gives an overview of the dynamic process migration mechanism, when the processes that run on different computing nodes have heavy I/O data dependency. The storage servers regularly compare the I/O access patterns of client computing processes. If two processes have similar access patterns, then the storage server triggers the migration of one process so it is placed near the process that has a similar access pattern. By improving the data locality, some of the I/O requests can be processed at the local file cache, which reduces the cost of I/O data transfer.

To put this mechanism to work, we propose a scheme of the Access Counting Diagram to profile the block access patterns of processes, which are used to estimate the pattern similarity. After that, a checkpoint/restart mechanism is employed to migrate a process, so the processes that have similar access patterns share the same computing node.

\subsection{Classifying Access Patterns}

We have designed a mechanism for characterizing the I/O access pattern of processes. This mechanism runs on the storage nodes that receive requests for blocks of data from clients file systems running on multiple nodes. Since a client file system can be solicited by multiple processes, we employ a piggybacking mechanism that has been implemented in our previous work [Liao et al. 2015] to characterize access patterns on a per-process basis. The sequences of I/O requests of each process are then stored as an Access Counting Diagram for further analysis.

3.1.1. Piggybacking Mechanism. Figure 2 illustrates the piggyback mechanism, in which the client file system is responsible for keeping extra information about the process and client file system. After that, it piggybacks the extra information with the relevant 


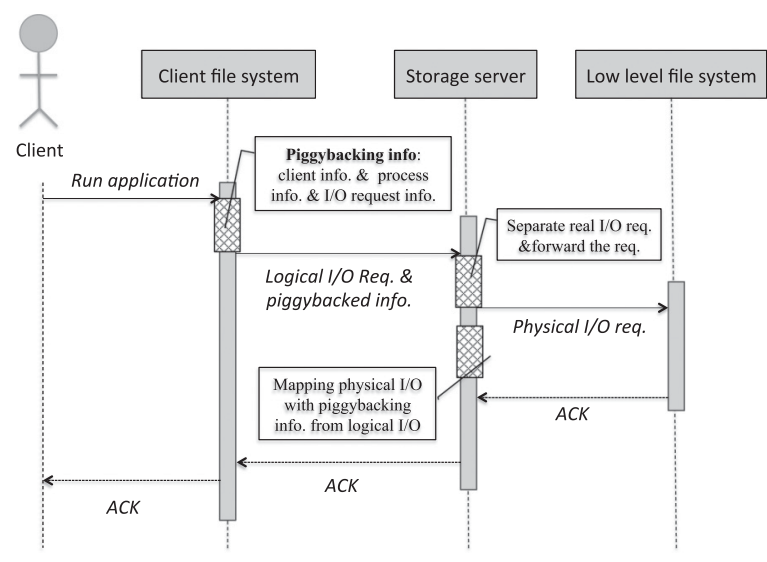

Fig. 2. Piggybacking mechanism for constructing mapping relationship between the logical I/O requests on client file systems and the physical I/O requests on storage servers with per-process granularity [Liao et al. 2015].

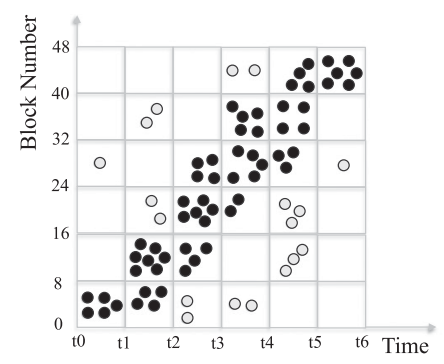

(a) Block Access Track

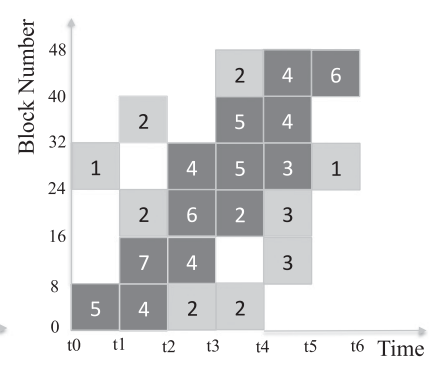

(b) Access Counting Diagram $(6 \times 6$ matrix $)$

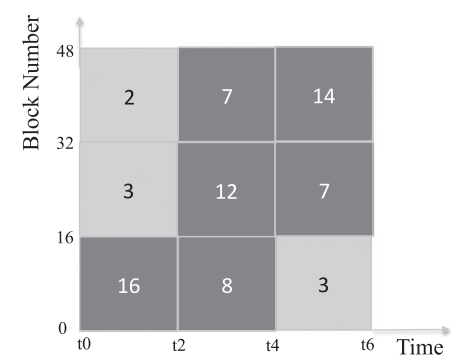

(C) N-compressed Access Counting Diagram $(\mathrm{N}=2)$ $(3 \times 3$ matrix $)$

Fig. 3. Classifying block access patterns by using the scheme of space-time diagram. (a) Forming a basic grid of space-time diagram to express the track of block access events; (b) counting the number of occurred block access in each grid element; (c) enlarging the size of grid element to reduce the number of grid elements.

client I/O request and then sends them to the corresponding storage server. On the other hand, the storage server parses the request to separate the piggybacked information and the real I/O request from the client request. In addition to forwarding the I/O request to the low-level file system, the storage server also records the disk I/O access event with the piggybacked information about the process identifier, and so on. As a result, the storage server makes a record for each block access event along with the information piggybacked by the corresponding client $\mathrm{I} / \mathrm{O}$ request, and then it is possible to profile block access patterns belonging to a specific process. ${ }^{1}$

3.1.2. Profiling Block Access Patterns. To model the block access pattern for a specific process and then benefit to matching patterns exactly and quickly, we propose an approach using a space-time diagram called the Access Counting Diagram. Both temporal and spatial correlations of block access events have been taken into consideration in this approach. Figure 3 illustrates the basic idea of the proposed space-time diagram for profiling block access patterns. In all sub-figures, the $\mathrm{X}$-axis indicates the time periods split with a fixed interval; and the Y-axis represents the range of accessed

\footnotetext{
${ }^{1}$ Note that the piggybacking mechanism is not a new idea. It has been proposed in our previous work [Liao et al. 2015], but it is a key technique to design and implement the newly proposed mechanism.
} 
blocks. All the access tracks that occurred in the time window ( $t 0$ to $t 6$ in the figure) are represented as points in Figure 3(a). Then, Figure 3(b) counts the block access events in each grid, and a two-dimensional array, that is, a matrix is used to represent the access pattern relevant to a process in the designated time period, that is, time window.

Because a process may access a huge amount of blocks, the size of the pattern array may become quite large. To reduce the size of the pattern array for quickly matching the block access patterns, we propose the $N$-compressed counting scheme, which can coarsen $N \times N$ grids into a big one and sum the values in the grids. Figure 3(c) shows an example of the 2-compressed counting conversion of Figure 3(b). In this case, the number of elements in the matrix is reduced from 36 to 9 . As a result, the time needed for matching patterns is decreased to a great extent, though it may introduce less accuracy in pattern matching. Section 4 discusses the impact of scaling the value of $N$ in the compressed counting scheme.

\subsection{Computing Access Pattern Similarity}

For estimating the degree of data dependency of two processes running on different computing nodes, we introduce the notion of pattern similarity. Equation (1) summarizes the algorithm that computes the similarity score of two block access patterns. The patterns are represented as two matrices having $m \times n$ elements (i.e., $m$ time intervals and $n$ block ranges). A similarity score close to 1.0 indicates a high similarity and that the two processes frequently access the same blocks of data,

$$
\mu=f\left(A_{m \times n}, B_{m \times n}\right)=1-\frac{\sum_{i=0}^{m-1}\left(\sum_{j=0}^{n-1} \frac{\left|A_{i, j}-B_{i, j}\right|}{\text { Maxevent }}\right)}{m \times n},
$$

where $A_{m \times n}$ and $B_{m \times n}$ mean the two involved matrices; $\left|A_{i, j}-B_{i, j}\right|$ represents the absolute difference of two integers, that is, $A_{i, j}$ and $B_{i, j}$, which are two corresponding elements in the different matrices; and Max event is a pre-defined integer value and set as the maximum number of block access events in the grid element by default.

As mentioned before, we keep the original access counting diagram and the compressed access counting diagram to compare the access pattern of processes. Therefore, the comparison algorithm first computes the similarity scores of the coarse-grained diagrams in order to promptly detect and discard the processes that have dissimilar access patterns. The fine-grained similarity is then computed only if the coarse-grained similarity is greater than a pre-defined threshold. The default value of the similarity threshold is fixed at 0.90 . To put it in other words, there are two steps in calculating the access pattern similarity:

—Step 1: The algorithm computes the similarity between two compressed access counting matrices. If the value of $\mu$ is larger than a pre-defined threshold, that is, a similarity threshold, then it continues to Step 2 for a fine-grained check. Otherwise, it stops and returns false, which means the result of "no similarity."

—Step 2: The algorithm conducts the pattern similarity computation between two original access counting matrices. When the value of $\mu$ is greater than the predefined threshold, it stops and returns true, which indicates the result of "having similarity"; otherwise, it returns false, which stands for "no similarity."

\subsection{Dynamic Process Migration}

This section discusses the details about dynamic process migration by using the MPI communication facility, as well as the checkpoint/restart mechanism.

3.3.1. Triggering Migration. When a storage server detects two processes having similar block access patterns, it triggers the migration of a process for grouping the processes on 
the same computing node. Consequently, the two processes can use the local file cache on the client file system for exchanging intermediate data rather than pulling/pushing the data from/to the storage nodes.

For the purpose of selecting a process to be migrated and choosing the destination node for the migrated process, the storage server first summaries the patterns' scales, according to Equation (2). These scales aggregate the number of I/O requests that were issued by a process over a period of time. Then, the storage server calculates the pattern similarity between the pattern having the largest scale and the patterns of the other processes running on other computing nodes according to Equation (1). If the storage server does not find a matched one, then the process having the second-largest scale is selected and the server searches for a process that matches it. This algorithm is repeated until all the processes have been traversed. As a result, the found process(es) are migrated to the destination computing node(s),

$$
\epsilon=f\left(A_{m \times n}\right)=\sum_{i=0}^{m-1} \sum_{j=0}^{n-1} A_{i, j},
$$

where $A_{m \times n}$ represents the matrix of access pattern and $A_{i, j}$ is the matrix element.

Certain policies can be used for exploring the process to be migrated. For instance, periodical scanning conducted by the storage server tries to find the target processes to be migrated, and this policy is currently used in our design.

We have also studied the time complexity of the proposed matching algorithm for a process-pair: It creates a time-space counting graph, that is, the matrix having a fixed number of grid elements. Then it computes the similarity for two matrices, which indicates its time complexity is $O(1)$, because the number of elements in the matrix is a constant due to configuration. Consider that we have a total of $n$ processes of application on the computing nodes; the newly proposed scheme will require $O\left(n^{2}\right)$ matching operations to find the targeted migration process(es) in a round of routine scans on the storage node.

However, the time complexity of $O\left(n^{2}\right)$ cannot work effectively for large-scale applications, which may have more than thousands of processes. To address this issue, we provide a configurable policy to compare only $m$ (a configurable variable of $m<<n$ ) processes, which have heavy I/O communications with the storage servers, for discovering processes to be migrated. That is because migrating the processes having fewer I/O workloads cannot benefit I/O system performance as expected. As a result, the proposed matching algorithm with the configurable policy needs $O\left(n \log n+m^{2}\right)$ operations, and the first part is needed for sorting the processes according to their total numbers of block access events in the specified period (i.e., pattern's scales); the second part is required for finding a matched process among $m$ selected processes. In brief, we conclude that when $m$ is much smaller than $n$, the expected time complexity of the proposed mechanism is $O(n \log n)$.

3.3.2. Migrating Process. The checkpoint/restart facility is utilized to perform the process migration. Figure 4 shows the interaction between the two involved computing nodes for completing the migration.

On the source computing node, the client file system first sends a message for building a connection state with the destination node, so both nodes are ready for conducting the process migration. Then, the client file system sends the consistent image of the targeted process after certain synchronization operations. On the destination computing node, the client file system restarts the stopped process when it has received the image of the targeted process. Consequently, the migrated process can continue running on the destination computing node and is able to use the local file cache on the 
Source computing node Destination computing node

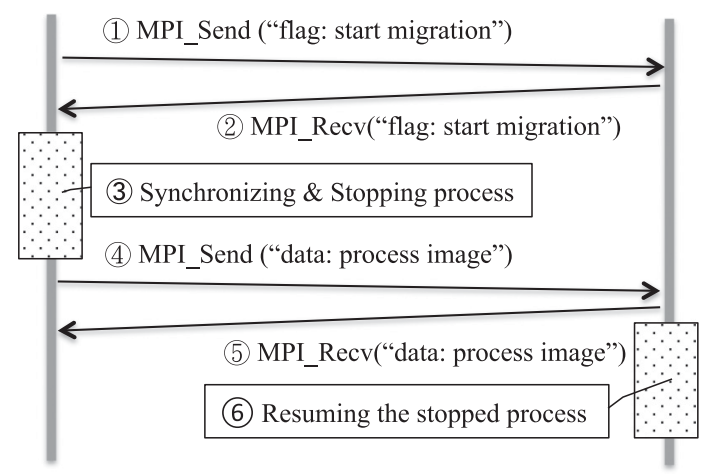

Fig. 4. The interaction between the source computing node and the destination computing node when performing a task of dynamic process migration.

node for exchanging data with other processes running on the same node, which have the similar access pattern.

Note that the migration requires that the destination node has enough memory for hosting the migrated process. Thus, we configured the file system to abandon the migration when the size of the checkpointed state is bigger than a pre-defined threshold, for example, one fourth of the memory capacity in our experiments.

\subsection{Implementation}

Regarding the functionality of process migration, we have integrated the MPICH2 library [MPI 2012] with the transparent kernel-level checkpoint/restart library implemented in our previous work [Liao 2012] after certain modifications on the checkpoint/restart library, according to the idea presented in Wang et al. [2008]. Thus, we can save the consistent state of the process running on the source node, and transfer the process image to the destination computing node by using MPI communication facilities, in order to resume the execution of the migrated process.

Moreover, we have implemented the newly proposed mechanism in the PARTE file system, which has been implemented from scratch in $\mathrm{C}$ and runs in the Linux environment [Liao and Ishikawa 2012]. The implementation has three modules running at the user level:

- partmds running on a specified server node. The module of active metadata server, which offers metadata services for client file systems and storage servers.

- parteost running on the storage nodes. The module of storage server that manages real file data. Moreover, it is in charge of profiling block access patterns, computing pattern similarity, and issuing the command of process migration periodically.

- partecfs running on the computing nodes. The module of the client file system has been designed and implemented based on FUSE [FUS 2010]. The client file system supports caching and managing for the cached data by employing the least recently used (LRU) policy. Furthermore, the client file system calls the checkpoint/restart library to fulfill dynamic process migration, after receiving the commands from the storage servers.

\section{EXPERIMENTS AND EVALUATION}

This section describes the experimental methodology for evaluating the proposed migration mechanism and then presents the experimental results. 
Table I. Specification of Nodes on the Cluster I and Cluster II

\begin{tabular}{l|ll}
\hline & Cluster I & Cluster II \\
\hline CPU & 4xIntel(R) E5410 2.33G & Intel(R) E5800 3.20G \\
\hline Memory & 1x4GB 1066MHz/DDR3 & 4GB DDR3-SDRAM \\
\hline Disk & 6x114GB 7200rpm SATA & 500GB 7200rpm SATA \\
\hline Network & Intel 82598EB, 10GbE & 10GbE \\
\hline OS & Ubuntu 13.10 & Debian 6.0.4 \\
\hline$\#$ of Nodes & 5 & 16 \\
\hline
\end{tabular}

\subsection{Experimental Setup}

4.1.1. Experimental Platform. Two clusters were used for carrying out the experiments: Tthe active metadata server and four storage servers were deployed on the 5 nodes of Cluster I, and all the client file systems were located on the 16 computing nodes of Cluster II. Both clusters are equipped with MPICH2-1.4.1, and connected with a 10GigE Ethernet. Table I shows the specifications of the nodes of both clusters.

4.1.2. Comparison Counterparts and Benchmarks. Because there is no related work that migrates ongoing processes on the basis of I/O workloads, we used the PARTE distributed file system with the following three configurations to run the benchmarks:

-Pattern-Based Migration, which is the distributed file system equipped with the proposed mechanism. It supports dynamic process migration according to the block access patterns. At first, the processes are allocated to the computing nodes by following the fashion of round-robin. But the processes can be migrated to other computing nodes during the execution.

We run the Pattern-Based Migration scheme with different time windows. Namely, Time $_{\text {win }}$ is configured as 256 block access events or 512 block access events, we respectively label them as Pattern-Based Migration+256, which is used by default, and Pattern-Based Migration +512 .

-Non-Migration, which does not enable process migration. The processes are bound to the computing nodes according to the round-robin fashion, and they are never migrated to other nodes.

-Optimal Static Placement, which is similar to the Non-Migration scheme, as it does not support process migration. The processes are statically allocated to the computing nodes, on the basis of their data dependency, after analyzing the source codes of benchmarks [Mercier and Clet-Ortega 2009]. In other words, the processes in a process-pair are mapped to the same computing nodes to enable exchanging their data by using the local file cache.

Note that this scheme is not a general solution, as it must understand the data dependency among the processes, and the placement of processes never change during the life cycle. That requires the analyis of the source codes of applications or that the application users offers the dependency information. This comparison counterpart is leveraged to demonstrate the gain/loss in a global scale resulting from our proposed Pattern-Based Migration scheme. Because we have modified the source codes of $m p i$ io test to emulate varied data dependency among the processes, we use this scheme while executing the benchmark of the mpi-io test.

Moreover, several benchmarks and applications were selected to run on the abovementioned storage systems to evaluate the applicability of the proposed process migration mechanism. All the selected applications are scheduled to continuously run three rounds.

- mpi-io test, which is constructed on top of MPI I/O calls and used to gather timing information for reading from and writing to file(s) using a variety of I/O profile 
configurations [MPI-IO Test]. In this benchmark, each process writes a large chunk of data to a non-overlapping, non-interleaved region of a file and then reads it back. We used mpi-io test $v 1.00 .021$ and made two modifications to the code. First, in order to emulate the different processes having data dependencies, we have slightly modified this benchmark: Process number $N$ reads the data flushed by process number $N+i$ in the $i t h$ round of execution. Namely, the data dependency varies from different round of execution. The second modification involved enabling asynchronous I/O interface. That means a process can read the data; when another corresponding process has written a part of data, the read and write operations are performed in parallel.

-Scalable Synthetic Compact Applications (SSCA), which is a benchmark suite consisting of six benchmarks, including the simulations of real-world applications, such as Bioinformatics Optimal Pattern Matching, Graph Analysis, and Synthetic Aperture Radar Application. Since we focus on I/O performance, only the source code of SSCA \#3 (version 0.1) was used in our experiments, which has Sensor-Processing and Knowledge-Formation steps, and focuses on large block data transfers and memory accesses, and small I/O operations [Haney et al. 2005].

- SPEEDY-LETKF, which is a typical real-world coupled system including the SPEEDY model and the Local Ensemble Transform Kalman Filter (LETKF) model. To be specific, SPEEDY is a simplified Atmospheric General Circulation Model developed by Molteni [2003]. In this experiment, it is coupled with the LETKF, which is a state-of-the-art approach to data assimilation [Hunt et al. 2007]. LETKF reads the output data from SPEEDY and then carries out the data assimilation. After that, the output results of LETKF are read by SPEEDY as the input data for continuing the next cycle of simulation.

4.1.3. Parameter Settings. In the PARTE file system, the block size of the system is $64 \mathrm{~KB}$, and the size of local file cache on the computing node was configured to $64 \mathrm{MB}$ (thus, the client file system can cache up to 1024 blocks of data), for which the LRU replacement policy was used to replace cache blocks. The default value of Max event was the maximum value of counting numbers among all grids in the access counting diagram. We set the size of grid as $32 \times 8$, which means each grid may have a 32 -block range and there are 8 time intervals in a pattern (Figure 3 shows with $8 \times 6$ ). The value of $N$ in the compressed access counting diagram was set to 2 by default. For each process, we keep the latest three-block access patterns which are used for calculating the pattern similarity. In each round of scan, 1 process is fixed to be migrated when the corresponding process running on another computing node has been found. Besides, we compare all involved processes to explore a possible matched pair of processes in our evaluation experiments, since the total number of processes is not large in the tests.

There are also several pre-defined values for computing the pattern similarity in the experiments, according to the size of local file cache. The number of block access events, that is, the time window of Time ${ }_{\text {win }}$, is relevant to the size of the local file cache, we recommend to set the number between the range of $\left[\right.$ Size $_{\text {cache }} /$ Size $_{\text {block }}, 2 \times$

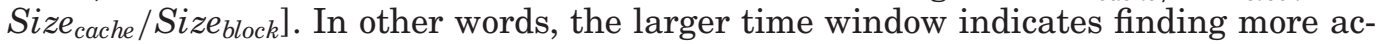
curate pattern similarity, and this can reduce the number of unnecessary migrations. However, it requires more time for estimating the similarity, and it might not perform process migrations, even when both processes have data dependency in reality. The storage servers calculated pattern similarity for the processes when they have completed $2 \times$ Time $_{\text {win }}$ block access events from the previous stage. The experiments of running mpi-io test, and SSCA \#3 have evaluated the proposed mechanism having different values of Time win $_{\text {. }}$ 


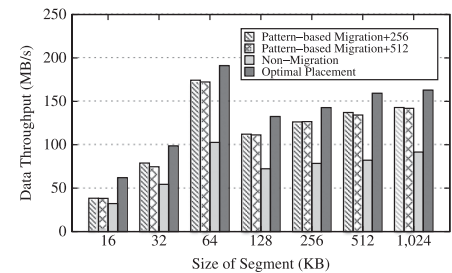

(a) Round 1: Read Data Throughput

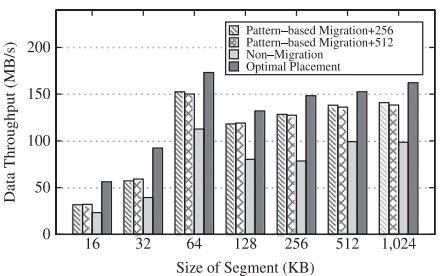

(d) Round 1: Write Data Throughput

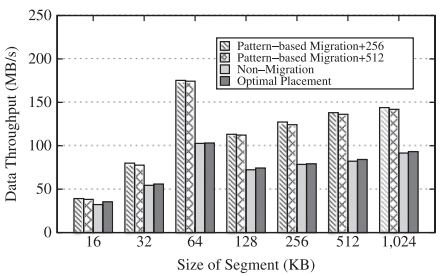

(b) Round 2: Read Data Throughput

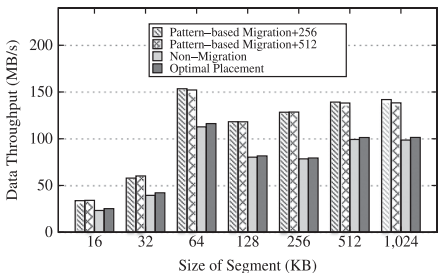

(e) Round 2: Write Data Throughput

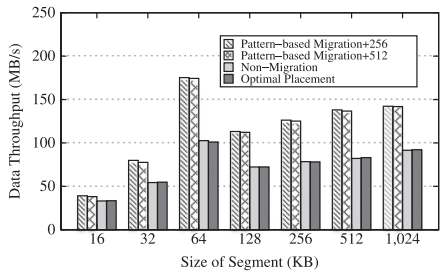

(c) Round 3: Read Data Throughput

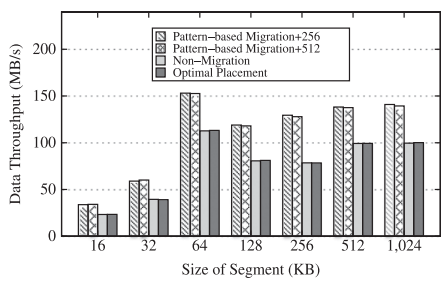

(f) Round 3: Write Data Throughput

Fig. 5. Data throughput obtained for the mpi-io benchmark with varying sizes of segments, when there are 16 client nodes. ((a), (b), and (c)) Read data throughput in the different rounds of execution; ((d), (e), and (f)) Write data throughput in the different rounds of execution.

Furthermore, in the case study of real-world application, we have also changed the value of the pre-defined similarity threshold, the value of $N$ for the compressed access counting scheme, and the number of block access events used for profiling the access pattern, respectively, in the case study. Because we conducted the experiments when the Time $_{\text {win }}$ are 256 and 512, respectively, but the results are almost same, we set the value of time window as 256 by default in the case study. The experimental results have illustrated the effectiveness with different level of triggers, and relevant results are reported in this section, as well.

\subsection{Experimental Results}

This section aims to disclose the effectiveness of the proposed mechanism, through running the selected benchmarks, as well as SPEEDY-LETKF, the real-world coupled application.

4.2.1. Results of mpi-io Test. In our tests, we executed the benchmark with 64 MPI processes (4 MPI processes per computing node) that read or write one shared 16GB file. That is to say, each process $p_{i}$ accesses the $(i+16 j)$ th segment at call $j$, for $0 \leq i \leq 15$, generating a fully sequential access pattern. Since the processes read the pieces of data written by the different processes in the different rounds of execution, we present the experimental results in the different rounds, separately. Figures 5(a), (b), and (c) present the experimental results of read data throughput, and Figures 5(d), (e), and (f) report the write data throughput in the different rounds of execution, when the size of segment varies from each other, and the number of client nodes is fixed as 16 (i.e., 64 MPI processes). In the same way, Figures 6(a)-(f) show the I/O data rate, when the number of client nodes varies from 1 node to 16 nodes, but the size of the segment is fixed at $64 \mathrm{~KB}$.

The experimental results show that the newly proposed mechanism improves the $\mathrm{I} / \mathrm{O}$ data rate by $14.3-76.6 \%$, in contrast to the Non-Migration scheme, because the proposed mechanism of block access pattern-based process migration can indeed dynamically reallocate the processes to the client nodes, on which there are processes 


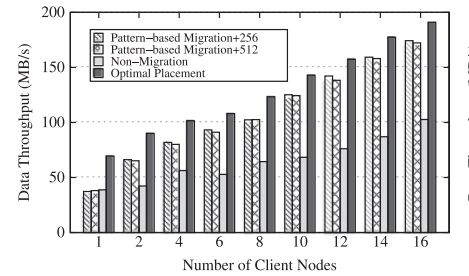

(a) Round 1: Read Data Throughput

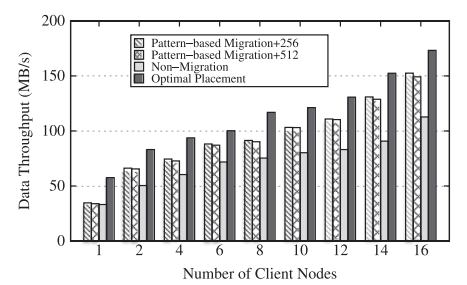

(d) Round 1: Write Data Throughput

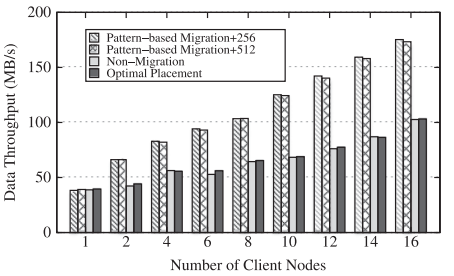

(b) Round 2: Read Data Throughput

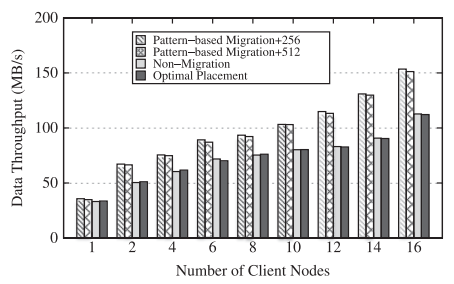

(e) Round 2: Write Data Throughput

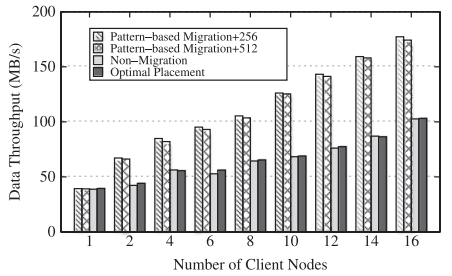

(c) Round 3: Read Data Throughput

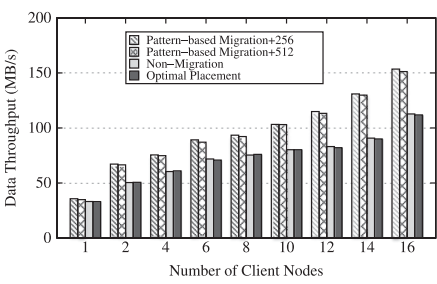

(f) Round 3: Write Data Throughput

Fig. 6. The data throughput with varying number of client nodes, when the size of segment is 64KB. ((a), (b), and (c)) Read data throughput in the different rounds of execution; ((d), (e), and (f)) write data throughput in the different rounds of execution.

with similar access patterns. Consequently, the processes are able to exchange their data by using the local file cache, instead of accessing the data through a storage server.

Further, the results reported in Figures 5 and 6 confirm that Pattern-Based Migration works well when the access pattern changes during the execution. In the first round, the Optimal Placement scheme (that binds optimally the processes for the first round) outperforms the Pattern-Based Migration scheme and Non-Migration (that placees the processes in the round-robin fashion). This is because, for the first round, the Optimal Placement binds the processes according to their data dependency, and the processes can exchange their data through the local file cache. Although the Pattern-Based Migration can dynamically migrate the processes for the same purpose, the migration causes an overhead that prevents this strategy from achieving the same performance as the Optimal Placement strategy. On the other hand, when the access pattern has changed in the second round and the third round of execution, the Optimal Static Placement scheme works like Non-Migration, and the processes have to use the global file system for exchanging their data. For these rounds, the Pattern-Based Migration strategy is able to dynamically adapt the placement of processes, and it achieves the same performance as for the first round. Pattern-Based Migration thus outperforms the other strategies by up to around $70 \%$.

Another interesting clue revealed in Figures 5 and 6, is that Pattern-Based Migration +256 slightly outperforms Pattern-Based Migration+512. This is because the smaller time window indicates less time for disclosing a matched process pair, and then the process migration can be conducted a little earlier in our selected benchmark. However, it is possible to perform incorrect migrations when the time window is too small, even though this did not occur in our experiments.

4.2.2. Results of SSCA \#3. In this series of experiments, the benchmark was configured with a scale factor of 6 and a dialed grid size of $4 \times 4 \times 4=64$ images on a single computing node, using the shared file system for storing the data. The size of the formed image is $1492 \times 2286$ Bytes, which means every node has to process $208 \mathrm{MB}$ of data, and all 16 client nodes run the same jobs. We did not analyze the source codes of SSCA \#3, so the Optimal Placement scheme has not been used in this experiment. 


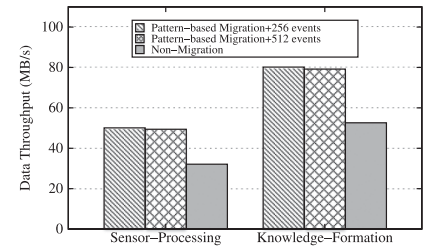

(a) Read Date Throughput

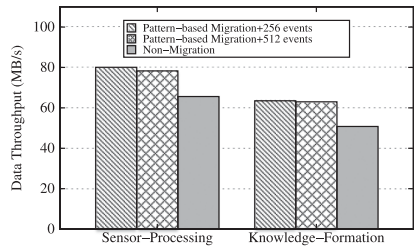

(b) Write Data Throughput

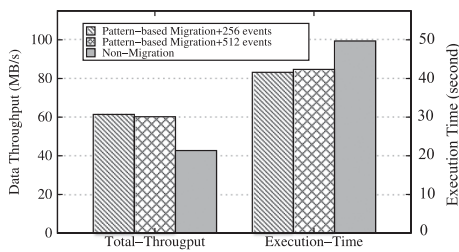

(c) Overall System Performance

Fig. 7. Results of running the HPCS SSCA \#3 benchmark. (a) Read data bandwidth in different steps; (b) Write data bandwidth in different steps; (c) Overall system performance in the measurements of total data throughput and the execution time.

Since the SSCA \#3 benchmark does not change its access pattern in the different rounds of execution, the average statistics over the three rounds of execution are reported. Figures 7(a) and (b) reports the read data throughput and write data throughput that were measured during the execution. Figure 7(c) presents the system performance by the measurements of the overall data throughput and the total execution time. From the reported results, we conclude that the proposed scheme of block access pattern-based process migration can effectively enhance the $\mathrm{I} / \mathrm{O}$ data throughput by $42.7 \%$ compared to Non-Migration and reduce the application runtime time by $14.8 \%$. Besides, note that the two selected sizes of the time window adopted by the proposed mechanism do not make any significant performance difference in this experiment.

4.2.3. Case Study of SPEEDY-LETKF. Because the first cycle of the SPEEDY-LETKF is the initialization cycle, and the access pattern does not change during the different cycles of execution, we run it with two cycles in each test, and we only report the results of the second cycle. In the tests, we set each LETKF ensemble member to have 16 parallel processes. That indicates that the number of SPEEDY processes is the number of LETKF ensemble members multiplied by 16 . The SPEEDY processes are initially allocated from client node 0 to 15 , following the round-robin, and the LETKF processes are placed from client node 15 to node 0 , following the round-robin, as well. The SPEEDY model executes 1-month integration and outputs the simulation results after the computation. The size of input data for the SPEEDY model is around 4.1MB, and the size of its output data, which is then read by the LETKF model, is 12.6MB. Furthermore, in order to explore the impact of different similarity thresholds and the value of $N$ in the compressed matrix, on the system performance, we have also executed the benchmark with diverse settings of these parameters. As Pattern-Based Migration +256 and Pattern-Based Migration +512 have yielded the similar results in our experiments, only Pattern-Based Migration +256 has been used in this case study and was labeled as Pattern-Based Migration by default. In this case study, we first measured the time required for running the application; next, we analyze the breakdown time; and, last, the results in terms of I/O data throughput are presented.

Time required for running the application. We measured the time required for executing the SPEEDY-LETKF application using the Pattern-Based Migration and Non-Migration strategies in the storage system. In the experiments, we scaled the number of ensemble LETKF from 1 to 10 , which indicates there are totally 16 to 160 processes for each kind of model. Figure 8 reports the time consumed for running the application benchmark utilizing the aforementioned two schemes. In particular, we employed the different scales of similarity and the value of $N$ in the Compressed Access Counting scheme. As the figure shows, the proposed mechanism can significantly reduce the time needed for running the application, compared to the Non-Migration mechanism. 


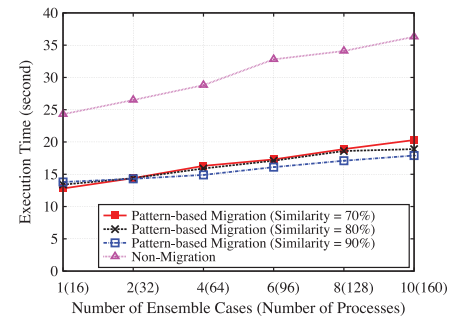

(a) Execution Time $(\mathrm{N}=2)$

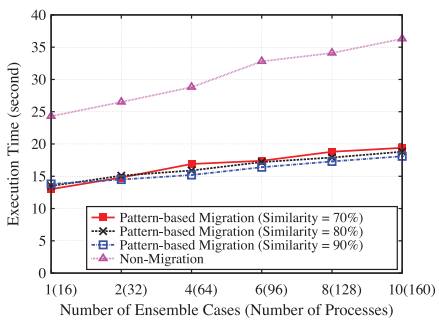

(b) Execution Time $(\mathrm{N}=4)$

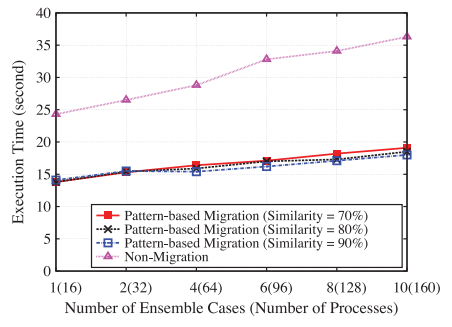

(c) Execution Time $(\mathrm{N}=6)$

Fig. 8. The total execution time when utilizing Pattern-Based Migration and Non-Migration.

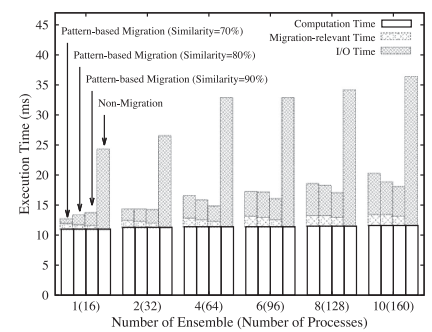

(a) Breakdown Time Analysis ( $\mathrm{N}=2)$

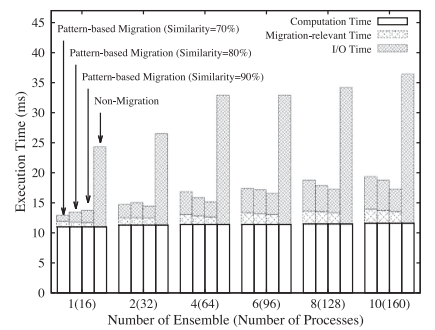

(b) Breakdown Time Analysis ( $=4)$

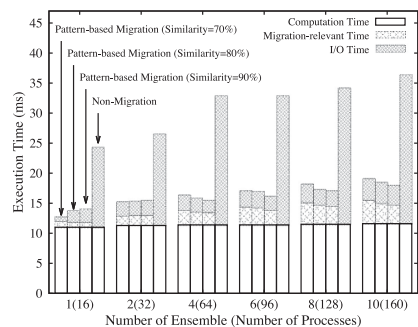

(c) Breakdown Time Analysis $(\mathrm{N}=6)$

Fig. 9. The breakdown time analysis when utilizing Pattern-Based Migration and Non-Migration.

In order to analyze more precisely the execution time of SPEEDY-LETKF, when using Pattern-Based Migration, we have conducted a breakdown analysis of the execution time. Figure 9 illustrates the results of time distribution. We conclude that the shorter execution time obtained when using Pattern-Based Migration is due to the I/O operations that are performed faster since the time required by computation remains the same. For example, when the size of ensemble instances is 10 (160 processes in total) in the test case, Pattern-Based Migration only needs around a quarter of the I/O time consumed by Non-Migration to perform the pattern-based migrations, as well as the requested I/O operations.

Another interesting clue shown in Figures 8 and 9 is the impact of the similarity threshold, and the different value of $N$ in the Compressed Access Counting scheme. Although varying $N$ does not impact the performance significantly in the evaluation, the different scales of similarity threshold indeed play a role in performance fluctuations. When there are few processes, lower similarity thresholds achieve the best performance, whereas the performance obtained with higher similarity thresholds is improved as the number of processes grows. This is because the lower scale of similarity threshold requires less time for finding a matched pattern but has a higher probability to migrate the process to the node that is not be the best choice. Therefore, it may work well when there are only a few processes, but the results are not that good when there are a large number of processes.

I/O Data Throughput. We have also measured the I/O data throughput when running the test case. Figure 10 shows the data throughput of the two selected comparison strategies. The results show that the Pattern-Based Migration strategy improves the I/O data throughput by up to $236.6 \%$ compared to the Non-Migration scheme.

In summary, SPEEDY-LETKF communicates between processes in a pairwise fashion that indicates that the data written by a SPEEDY process is then read by the corresponding LETKF process. Consequently, the time required for exchanging the data between two kinds of processes can be remarkably reduced and the data rate 


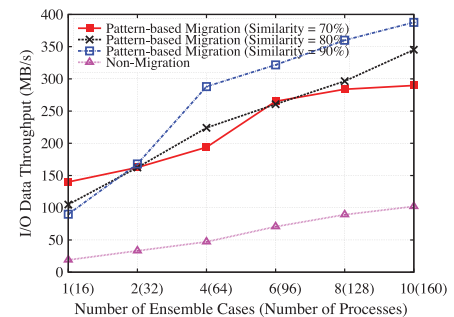

(a) I/O Data Throughput $(\mathrm{N}=2)$

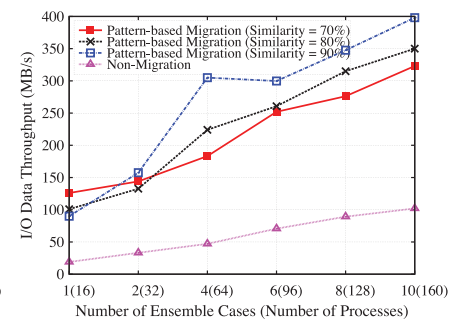

(b) I/O Data Throughput $(\mathrm{N}=4)$

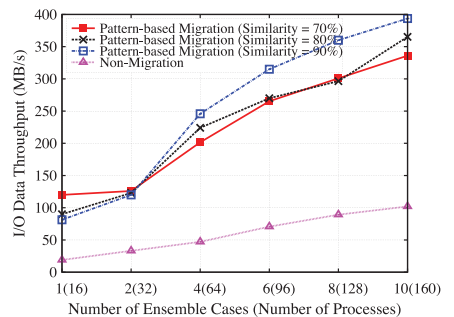

(c) I/O Data Throughput $(\mathrm{N}=6)$

Fig. 10. I/O data throughput when utilizing Pattern-Based Migration and Non-Migration.

Table II. Process Migration Overhead

\begin{tabular}{lccc}
\hline Benchmark & \# of Migrations & Traffic (MB) & Time (ms) \\
\hline mpi-io_test & 31 & 1400 & 1298 \\
SSCA \#3 & 7 & 742 & 1002 \\
SPEEDY-LETKF (16) & 8 & 422 & 1211 \\
SPEEDY-LETKF (32) & 16 & 825 & 1473 \\
SPEEDY-LETKF (64) & 32 & 1613 & 1794 \\
SPEEDY-LETKF (96) & 49 & 2211 & 2172 \\
SPEEDY-LETKF (128) & 65 & 2764 & 2445 \\
SPEEDY-LETKF (160) & 81 & 3187 & 2667 \\
\hline
\end{tabular}

can be significantly improved, when both of them are allocated in the same computing node, and the local file cache on the node is used for data exchange.

4.2.4. Overhead Analysis. The evaluation has shown that the pattern-based dynamical process migration can effectively and practically improve the performance for different workloads, but it is also necessary to measure the overhead caused the migration scheme we propose. In fact, in addition to conducing the process migration, the proposed mechanism also needs to analyze the block access events to profile the patterns, and computing the pattern similarity for triggering migration. This section evaluates the overhead caused by the migration of the processes between computing nodes, as well as the overhead.

Overhead on process migration. Table II reports the overhead caused by the migration of processes, when running the benchmarks with default settings. Namely, the value of Time $_{w i n}$ is 256 block access events, and the value of Similarity is 0.9 . Because the processes in the selected benchmarks have heavy data dependency, nearly half the processes have been migrated to other nodes. However, the results show that the overhead of the migration overhead is not high. For instance, SPEEDY-LETKF running 160 processes in total consumed 2.7s of downtime for process migrations, but the migration saved more than 18 s of $\mathrm{I} / \mathrm{O}$ time.

While traditional checkpoint/restart mechanisms rely on disk operations, the Pattern-Based migration transfers the state of a process to the destination client node using the MPI communication facility. As a result, the process can be resumed to execute on the destination node with a short time interruption. In other words, the downtime of the migrated process is reduced when using MPI for transferring the process state. This downtime is compensated with the I/O operations that are carried out faster, which was shown in previous sections.

Another interesting observation is that while the number of processes becomes larger, more migration overhead is required. We believe that this is due to transferring more state data of the checkpointed processes and restarting more migrated processes. 
Table III. Computation and Space Overhead

\begin{tabular}{lcc}
\hline Benchmark & Time (\%) & Space (MB) \\
\hline mpi-io_test & 2.18 & 169.64 \\
SSCA \#3 & 1.25 & 26.98 \\
SPEEDY-LETKF (16) & 1.87 & 10.21 \\
SPEEDY-LETKF (32) & 1.81 & 22.18 \\
SPEEDY-LETKF (64) & 1.78 & 30.45 \\
SPEEDY-LETKF (96) & 1.76 & 42.16 \\
SPEEDY-LETKF (128) & 1.78 & 60.22 \\
SPEEDY-LETKF (160) & 1.77 & 77.44 \\
\hline
\end{tabular}

Overhead on computation and storage. The Pattern-Based Migration mechanism records the block access events and then profiles block access patterns using the Access Counting Scheme in order to calculate the pattern similarity and to direct the process migration. Table III reports the overhead on computation and space storage due to the Access Counting Scheme for the benchmarks presented in the previous sections.

The results show that the overhead in terms of computation is minor. The overhead in terms of space storage remains low: Less than 200MB of disk space is used for storing trace logs. Thus, analyzing the similarity of block access patterns and activating the process migration can be done on the same machine as the storage system without causing too much computation and disk overhead. For long-time running applications, the size of trace logs may become extraordinarily large; in this case, the Pattern-Based Migration scheme may discard certain logs that occurred early, because disk block correlations are relatively stable during certain period, and the disk access logs at earlier stages do not reflect the current access patterns [Li et al. 2004].

\section{CONCLUSION}

This article has proposed, implemented, and evaluated a novel process migration mechanism for yielding better I/O performance. It leverages the block access pattern similarity of processes, which is computed by the storage servers, to properly perform migrations. The experiments have shown that this mechanism can benefit coupled applications or other scientific applications running on high-performance clusters without any modifications to the application themselves. In other words, we first profile the block access pattern of processes for a specified period using the scheme of the Access Counting Diagram. Next, we calculate the pattern similarity of the patterns of process pairs and search for processes that have similar access patterns. When such a pair of processes is found, one of the processes is migrated to the other process computing node. Therefore, both processes can exchange data via the local file cache on the computing node instead of using the global file system. The results of the evaluation experiments show that not only the I/O data throughput can be remarkably improved but also the execution time of the application can be greatly reduced.

We also emphasize that the idea of process migration according to pattern similarity presented in this article can be applied to other conventional distributed/parallel file systems such as Lustre, the Google file system, the Parallel Virtual File System, or the Hadoop distributed file system. Our current implementation selects the targeted migration process on the basis of I/O workloads, and the computing workloads are not considered. This may result in migrating an I/O-bound process running on an idle computing node to a busy one, although this did not appear in the evaluation experiments. Moreover, the intra-application communications among processes are not taken into account before conducting process migrations. Therefore, we intend to optimize the 
implementation by using some MPI profiling tools to gather the information about the computing utilization on the computing nodes and the intra-application communication traffics to properly carry out process migrations. Besides, intelligently adapting the values of parameters used in the proposed mechanism for the different application contexts is another direction of our future work.

\section{REFERENCES}

2010. Filesystem in Userspace (FUSE). http://fuse.sourceforge.net. (2010). Online; accessed Nov. 2010.

2012. MPICH2. https://www.mpich.org. (2012). Online; Accessed in Dec., 2012.

2014. MPI-IO Test (fs test). http://institute.lanl.gov/data/software/. (2014). Online; Accessed in May, 2014.

D. H. Bailey, E. Barszcz, J. T. Barton, D. S. Browning, R. L. Carter, L. Dagum, R. A. Fatoohi, P. O. Frederickson, T. A. Lasinski, R. S. Schreiber, H. D. Simon, V. Venkatakrishnan, and S. K. Weeratunga. 1991. The NAS parallel benchmarks\&Mdash;summary and preliminary results. In Proceedings of the 1991 ACM/IEEE Conference on Supercomputing (Supercomputing'91). ACM, New York, NY, 158-165. DOI :http://dx.doi. org/10.1145/125826.125925

Hu Chen, Wenguang Chen, Jian Huang, Bob Robert, and H. Kuhn. 2006. MPIPP: An automatic profileguided parallel process placement toolset for SMP clusters and multiclusters. In Proceedings of the 20th Annual International Conference on Supercomputing (ICS'06). ACM, New York, NY, 353-360. DOI :http://dx.doi.org/10.1145/1183401.1183451

Quan Chen and Minyi Guo. 2014. Adaptive workload-aware task scheduling for single-ISA asymmetric multicore architectures. ACM Trans. Archit. Code Optim. 11, 1, Article 8 (Feb. 2014), 25 pages. DOI : http://dx.doi.org/10.1145/2579674

Luwei Cheng and Cho-Li Wang. 2012. vBalance: Using interrupt load balance to improve I/O performance for SMP virtual machines. In Proceedings of the Third ACM Symposium on Cloud Computing (SoCC'12). ACM, New York, NY, Article 2, 14 pages. DOI :http://dx.doi.org/10.1145/2391229.2391231

Christopher Clark, Keir Fraser, Steven Hand, Jacob Gorm Hansen, Eric Jul, Christian Limpach, Ian Pratt, and Andrew Warfield. 2005. Live migration of virtual machines. In Proceedings of the 2nd Conference on Symposium on Networked Systems Design \& Implementation-Volume 2 (NSDI'05). USENIX Association, Berkeley, CA, 273-286. http://dl.acm.org/citation.cfm?id=1251203.1251223.

Lauro Beltrão Costa, Samer Al-Kiswany, Hao Yang, and Matei Ripeanu. 2014. Supporting storage configuration for I/O intensive workflows. In Proceedings of the 28th ACM International Conference on Supercomputing (ICS'14). ACM, New York, NY, 191-200. DOI: http://dx.doi.org/10.1145/2597652.2597679

Jeffrey Dean and Sanjay Ghemawat. 2008. MapReduce: Simplified data processing on large clusters. Commun. ACM 51, 1 (Jan. 2008), 107-113. DOI :http://dx.doi.org/10.1145/1327452.1327492

Xiaoning Ding, Song Jiang, Feng Chen, Kei Davis, and Xiaodong Zhang. 2007. DiskSeen: Exploiting disk layout and access history to enhance I/O prefetch. In 2007 USENIX Annual Technical Conference on Proceedings of the USENIX Annual Technical Conference (ATC'07). USENIX Association, Berkeley, CA, USA, Article 20, 14 pages. http://dl.acm.org/citation.cfm?id=1364385.1364405.

Xiangyu Dong, Yuan Xie, Naveen Muralimanohar, and Norman P. Jouppi. 2011. Hybrid checkpointing using emerging nonvolatile memories for future exascale systems. ACM Trans. Archit. Code Optim. 8, 2, Article 6 (June 2011), 29 pages. DOI :http://dx.doi.org/10.1145/1970386.1970387

Jack Dongarra, Pete Beckman, Terry Moore, Patrick Aerts, Giovanni Aloisio, Jean-Claude Andre, David Barkai, Jean-Yves Berthou, Taisuke Boku, Bertrand Braunschweig, Franck Cappello, Barbara Chapman, Xuebin Chi, Alok Choudhary, Sudip Dosanjh, Thom Dunning, Sandro Fiore, Al Geist, Bill Gropp, Robert Harrison, Mark Hereld, Michael Heroux, Adolfy Hoisie, Koh Hotta, Zhong Jin, Yutaka Ishikawa, Fred Johnson, Sanjay Kale, Richard Kenway, David Keyes, Bill Kramer, Jesus Labarta, Alain Lichnewsky, Thomas Lippert, Bob Lucas, Barney Maccabe, Satoshi Matsuoka, Paul Messina, Peter Michielse, Bernd Mohr, Matthias S. Mueller, Wolfgang E. Nagel, Hiroshi Nakashima, Michael E. Papka, Dan Reed, Mitsuhisa Sato, Ed Seidel, John Shalf, David Skinner, Marc Snir, Thomas Sterling, Rick Stevens, Fred Streitz, Bob Sugar, Shinji Sumimoto, William Tang, John Taylor, Rajeev Thakur, Anne Trefethen, Mateo Valero, Aad Van Der Steen, Jeffrey Vetter, Peg Williams, Robert Wisniewski, and Kathy Yelick. 2011. The international exascale software project roadmap. Int. J. High Perform. Comput. Appl. 25, 1 (Feb. 2011), 3-60. DOI :http://dx.doi.org/10.1177/1094342010391989

Cong Du, Xian-He Sun, and Ming Wu. 2007. Dynamic scheduling with process migration. In Proceedings of the Seventh IEEE International Symposium on Cluster Computing and the Grid (CCGRID'07). IEEE Computer Society, Washington, DC, 92-99. DOI : http://dx.doi.org/10.1109/CCGRID.2007.46

Karl Fuerlinger, Nicholas J. Wright, and David Skinner. 2010. Effective performance measurement at petascale using IPM. In Proceedings of the 2010 IEEE 16th International Conference on Parallel 
and Distributed Systems (ICPADS'10). IEEE Computer Society, Washington, DC, 373-380. DOI :http:// dx.doi.org/10.1109/ICPADS.2010.16

Zhenhuan Gong. 2013. Multi-Level Data Layout Optimization for Heterogeneous Access Patterns. Ph.D. Dissertation. Advisor(s) Samatova, Nagiza F. AAI3586115.

James J. Hack, Julie M. Caron, G. Danabasoglu, Keith W. Oleson, Cecilia Bitz, and John E. Truesdale. 2006. CCSM-CAM3 climate simulation sensitivity to changes in horizontal resolution. J. Clim. 19, 11 (2006), 2267-2289.

Ryan Haney, Theresa Meuse, Jeremy Kepner, and James Lebak. 2005. The HPEC challenge benchmark suite. In HPEC 2005 Workshop.

Jun He, John Bent, Aaron Torres, Gary Grider, Garth Gibson, Carlos Maltzahn, and Xian-He Sun. 2013. I/O acceleration with pattern detection. In Proceedings of the 22nd International Symposium on HighPerformance Parallel and Distributed Computing (HPDC'13). ACM, New York, NY, 25-36. DOI:http:// dx.doi.org/10.1145/2462902.2462909

Brian R. Hunt, Eric J. Kostelich, and Istvan Szunyogh. 2007. Efficient data assimilation for spatiotemporal chaos: A local ensemble transform Kalman filter. Physica D 230, 1 (2007), 112-126.

Emmanuel Jeannot, Guillaume Mercier, and François Tessier. 2014. Process placement in multicore clusters: Algorithmic issues and practical techniques. IEEE Trans. Parallel Distrib. Syst. 25, 4 (2014), 993-1002.

Song Jiang, Xiaoning Ding, Yuehai Xu, and Kei Davis. 2013. A prefetching scheme exploiting both data layout and access history on disk. Trans. Storage 9, 3, Article 10 (Aug. 2013), 23 pages. DOI:http://dx.doi.org/ $10.1145 / 2508010$

Jay Larson, Robert Jacob, and Everest Ong. 2005. The model coupling toolkit: A new Fortran90 toolkit for building multiphysics parallel coupled models. Int. J. High Perform. Comput. Appl. 19, 3 (2005), $277-292$.

Zhenmin Li, Zhifeng Chen, Sudarshan M. Srinivasan, and Yuanyuan Zhou. 2004. C-miner: Mining block correlations in storage systems. In Proceedings of the 3rd USENIX Conference on File and Storage Technologies (FAST'04). USENIX Association, Berkeley, CA, USA, 173-186. http://dl.acm.org/ citation.cfm?id=1096673.1096695.

Jianwei Liao. 2012. A new concurrent checkpoint mechanism for embeded multi-core systems. Comput. Inform. 31, 3 (2012), 693-709.

Jianwei Liao and Yutaka Ishikawa. 2012. Partial replication of metadata to achieve high metadata availability in parallel file systems. In Proceedings of the 201241 st International Conference on Parallel Processing (ICPP'12). IEEE Computer Society, Washington, DC, 168-177. DOI:http://dx.doi.org/ 10.1109/ICPP.2012.49

Jianwei Liao, Francois Trahay, Balazs Gerofi, and Yutaka Ishikawa. Prefetching on storage servers through mining access patterns on blocks. IEEE Trans. Parallel Distrib. Syst. DOI:http://dx.doi.org/ 10.1109/TPDS.2015.2496595

Esteban Meneses, Xiang Ni, Gengbin Zheng, Celso L. Mendes, and Laxmikant V. Kale. 2015. Using migratable objects to enhance fault tolerance schemes in supercomputers. IEEE Trans. Parallel Distrib. Syst. 26, 7 (2015), 2061-2074.

Guillaume Mercier and Jérôme Clet-Ortega. 2009. Towards an efficient process placement policy for MPI applications in multicore environments. In Proceedings of the 16th European PVM/MPI Users' Group Meeting on Recent Advances in Parallel Virtual Machine and Message Passing Interface. Springer-Verlag, Berlin, 104-115. DOI : http://dx.doi.org/10.1007/978-3-642-03770-2_17.

Guillaume Mercier and Emmanuel Jeannot. 2011. Improving MPI applications performance on multicore clusters with rank reordering. In Proceedings of the 18th European MPI Users' Group Conference on Recent Advances in the Message Passing Interface (EuroMPI'11). Springer-Verlag, Berlin, 39-49. http://dl.acm.org/citation.cfm?id=2042476.2042483

Pierre Michaud, André Seznec, Damien Fetis, Yiannakis Sazeides, and Theofanis Constantinou. 2007. A study of thread migration in temperature-constrained multicores. ACM Trans. Archit. Code Optim. 4, 2, Article 9 (June 2007). DOI : http://dx.doi.org/10.1145/1250727.1250729

Franco Molteni. 2003. Atmospheric simulations using a GCM with simplified physical parametrizations. I: Model climatology and variability in multi-decadal experiments. Climate Dynam. 20, 2-3 (2003), 175191.

Juan Piernas, Jarek Nieplocha, and Evan J. Felix. 2007. Evaluation of active storage strategies for the lustre parallel file system. In Proceedings of the 2007 ACM/IEEE Conference on Supercomputing (SC'07). ACM, New York, NY, Article 28, 10 pages. DOI : http://dx.doi.org/10.1145/1362622.1362660

Juan Piernas-Canovas and Jarek Nieplocha. 2010. Implementation and evaluation of active storage in modern parallel file systems. Parallel Comput. 36, 1 (Jan. 2010), 26-47. DOI :http://dx.doi.org/10.1016/ j.parco.2009.11.002 
Laercio L. Pilla, Christiane Pousa Ribeiro, Daniel Cordeiro, Chao Mei, Abhinav Bhatele, Philippe O. A. Navaux, Francois Broquedis, Jean-Francois Mehaut, and Laxmikant V. Kale. 2012. A hierarchical approach for load balancing on parallel multi-core systems. In Proceedings of the 201241 st International Conference on Parallel Processing (ICPP'12). IEEE Computer Society, Washington, DC, 118-127. DOI : http://dx.doi.org/10.1109/ICPP.2012.9

Mustafa M. Tikir, Michael A. Laurenzano, Laura Carrington, and Allan Snavely. 2009. PSINS: An open source event tracer and execution simulator for MPI applications. In Euro-Par 2009 Parallel Processing. Springer, Berlin, 135-148.

S. Valcke, V. Balaji, A. Craig, C. DeLuca, R. Dunlap, R. W. Ford, R. Jacob, Jay Larson, R. O’Kuinghttons, G. D. Riley, and others. 2012. Coupling technologies for earth system modelling. Geosci. Model Develop. 5, 6 (2012), 1589-1596.

Sophie Valcke, Reinhard G. Budich, Mick Carter, Eric Guilyardi, Marie-Alice Foujols, Michael Lautenschlager, René Redler, Lois Steenman-Clark, and Nils Wedi. 2006. The PRISM software framework and the OASIS coupler. In Annual BMRC Modelling Workshop "The Australian Community Climate and Earth System Simulator (ACCESS)-Challenges and Opportunities."

Christian Vecchiola, Suraj Pandey, and Rajkumar Buyya. 2009. High-performance cloud computing: A view of scientific applications. In Proceedings of the 2009 10th International Symposium on Pervasive Systems, Algorithms, and Networks (ISPAN'09). IEEE Computer Society, Washington, DC, 4-16. DOI : http://dx.doi.org/10.1109/I-SPAN.2009.150

Chao Wang, Frank Mueller, Christian Engelmann, and Stephen L. Scott. 2008. Proactive process-level live migration in HPC environments. In Proceedings of the 2008 ACM/IEEE Conference on Supercomputing (SC'08). IEEE Press, Piscataway, NJ, Article 43, 12 pages. http://dl.acm.org/citation. $\mathrm{cfm}$ ?id=1413370.1413414.

Dan Williams, Hani Jamjoom, and Hakim Weatherspoon. 2012. The Xen-blanket: Virtualize once, run everywhere. In Proceedings of the 7th ACM European Conference on Computer Systems (EuroSys'12). ACM, New York, NY, 113-126. DOI : http://dx.doi.org/10.1145/2168836.2168849

Yulai Xie, Dan Feng, Yan Li, and Darrell D. E. Long. 2016. Oasis. Future Gener. Comput. Syst. 56, C (March 2016), 746-758. DOI : http://dx.doi.org/10.1016/j.future.2015.08.011

Fang Zheng, Hongbo Zou, Greg Eisenhauer, Karsten Schwan, Matthew Wolf, Jai Dayal, Tuan-Anh Nguyen, Jianting Cao, Hasan Abbasi, Scott Klasky, Norbert Podhorszki, and Hongfeng Yu. 2013. FlexIO: I/O middleware for location-flexible scientific data analytics. In Proceedings of the 2013 IEEE 27th International Symposium on Parallel and Distributed Processing (IPDPS'13). IEEE Computer Society, Washington, DC, 320-331. DOI : http://dx.doi.org/10.1109/IPDPS.2013.46

Received October 2015; revised March 2016; accepted March 2016 